Volume 70, Number 1, Pages 205215(2021)

DOI: $10.31801 /$ cfsuasmas.721513

ISSN 1303-5991 E-ISSN 2618-6470

Received by the editors: April 16, 2020; Accepted: December 18, 2020

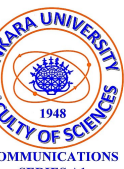

\title{
THE UNIFORM CONVERGENCE OF FOURIER SERIES EXPANSIONS OF A STURM-LIOUVILLE PROBLEM WITH BOUNDARY CONDITION WHICH CONTAINS THE EIGENPARAMETER
}

\author{
SERTAC GOKTAS ${ }^{1}$ and EMIR ALI MARIS ${ }^{2}$ \\ ${ }^{1}$ Department of Mathematics, Mersin University, Mersin, TURKEY \\ ${ }^{2}$ Vocational School of Technical Sciences, Mersin University, Mersin, TURKEY
}

\begin{abstract}
This paper is devoted to investigating the uniform convergence conditions of Fourier series expansions of continuous functions in terms of eigenfunctions of a Sturm-Liouville problem with eigenparameter in one of the boundary conditions on a closed interval. Such problems are quite common in mathematical physics problems.
\end{abstract}

\section{INTRODUCTION}

In many mathematical physics problems, partial differential equations are encountered. The most common of these are differential equations of the second order. Partial differential equations of the second order of the hyperbolic type occur most frequently in physical problems with vibration processes such as the transverse or longitudinal vibrations of a string, a membrane and rod, the vibrational energy of a string, hydrodynamics and acoustics, etc. 16. On the other hand, differential equations often give an infinite number of solutions. However, for the mathematical description of a physical process, sufficient conditions must be determined to uniquely determine the process. That is, solutions should be limited under certain conditions. Whence, it is necessary to supplement that equation with certain additional equations such as initial or boundary conditions in order to specify the process uniquely when the physical problem is led to a partial differential equation. In this way, a physical model corresponds to a boundary value problem.

2020 Mathematics Subject Classification. 34L05, 34L20, 34L10, 34B05.

Keywords and phrases. Eigenvalues, eigenfunctions, uniform convergence of spectral expansion.

\stcgoktas@gmail.com-Corresponding author; e.ali.maris@gmail.com

(D) 0000-0001-7842-6309; 0000-0001-7620-8754.

(C)2021 Ankara University Communications Faculty of Sciences University of Ankara-Series A1 Mathematics and Statistics 
One of the widest methods used to solve partial differential equations is Fourier's method, which is also called the method of separation of variables. Finding values $\lambda$, called eigenvalues for which non-trivial solutions called eigenfunctions, of the eigenvalue problem that arises with the application of this method is required, and also obtaining these solutions of it. The problem formulated in this way is also called Sturm-Liouville's problem. It is known from Fourier series theory that the arbitrary partially continuous and partially differentiable a function $f(x)$ given in the interval $[0, l]$ can be expanded as Fourier series in the sine and cosine functions or eigenfunctions of an eigenvalue problem. Therefore, the solutions of an eigenvalue problem are in the form of an infinite series. If this series divergent or the function defined by this series is not differentiable, then it can't naturally represent a solution of a differential equation. Consequently, investigating the convergence conditions of these series and finding the representation of the solutions are of great importance.

In this study, our aim is to investigate the uniform convergence conditions of spectral expansions of continuous functions in terms of eigenfunctions of the SturmLiouville problem:

$$
\begin{gathered}
-u^{\prime \prime}+q(x) u=\lambda u, \quad 0<x<1, \\
u(0)=0, \quad u^{\prime}(0)=\lambda\left(a u(1)+b u^{\prime}(1)\right)
\end{gathered}
$$

where $\lambda$ is an eigenparameter, $q(x) \in L_{1}(0,1)$ is a complex-valued function, $a$ and $b$ are arbitrary complex numbers which satisfy the condition $|a|+|b| \neq 0$.

In the spectral theory of differential operators, there are many articles containing Sturm-Liouville equations with boundary conditions linearly or polynomially dependent on the spectral parameter $66,7,3,4,5,1,2,9,10,11,12,17$, . The convergence conditions of Fourier series expansions of functions in some functional class of Sturm-Liouville operators are investigated in $6,7,7,3,4,5,1,2,9,10,0$. For example, the convergence conditions of series expansions of the following problems are studied in 1], 6], 7], respectively: spectral problems that appear modeling heat transfer in a homogeneous rod with a linear relation between the heat flux and temperature at one endpoint and with a lumped heat capacity at the other endpoint, spectral problems that appear in a model of a transrelaxation heat process and in the mathematical description of vibrations of a loaded string and, spectral problems that appear on vibrations of a homogeneous loaded string, torsional vibrations of a rod with a pulley at one end, heat propagation in a rod with lumped heat capacity at one end, and the current in a cable grounded at one end through a concentrated capacitance or inductance.

Moreover, the spectral problem

$$
\begin{gathered}
-u^{\prime \prime}+q(x) u=\lambda u, 0<x<1, \\
u(0)=0, u^{\prime}(0)-a \lambda u(1)=0
\end{gathered}
$$

was considered in 14 for $q(x)=0$ and $a>0$; in 8 for $q(x) \neq 0$ and $a \neq 0$. The conditions of the uniform convergence of spectral expansions of continuous functions in the system of eigenfunctions in these studies were established. Note 
that, the problems considered in these studies are special cases of the problem (1)-(2). Therefore, we will assume that $b \neq 0$ from now on.

\section{Preliminaries}

In this section, some properties will be given in order to reach the desired results of the problem (1)-(2).

Denote the solution of the equation (1), satisfying the initial conditions

$$
\psi(0)=0, \quad \psi^{\prime}(0)=1
$$

by $\psi(x)=\psi(x, \lambda)$.

Lemma 1 (13]). Let $\lambda=\mu^{2}$. Then,

$$
\psi(x, \lambda)=\frac{\sin \mu x}{\mu}+\frac{1}{\mu} \int_{0}^{x} \sin \{\mu(x-\theta)\} q(\theta) \psi(\theta, \lambda) d \theta .
$$

Lemma $2([13])$. Let $\mu=\sigma+i t$. Then, there exists $\mu_{0}>0$ such that, for $|\mu|>\mu_{0}$, the estimate

$$
\psi(x, \lambda)=\frac{\sin \mu x}{\mu}+O\left(\frac{e^{|t| x}}{|\mu|^{2}}\right)
$$

is valid, where the function $O\left(e^{|t| x}|\mu|^{-2}\right)$ is the entire function of $\mu$ for any fixed $x$ in $[0,1]$. Moreover, the estimate (5) is uniform with respect to $x \in[0,1]$.

Theorem 3 ( 15]). All the eigenvalues of the problem (1)-(2) are simple. Moreover, they have form infinite sequence $\lambda_{n}(n=0,1,2, \ldots)$ which has no finite limit points. And, the following asymptotic estimate are valid for sufficiently large $n$ :

$$
\begin{gathered}
\lambda_{n}=\left[\left(n-\frac{1}{2}\right) \pi\right]^{2}+O(1), \\
\psi_{n}(x)=\psi\left(x, \lambda_{n}\right)=\frac{\sin \left(n-\frac{1}{2}\right) \pi x}{n \pi}+O\left(\frac{1}{n^{2}}\right) .
\end{gathered}
$$

Theorem $4(15])$. If $\left(b \lambda_{n}\right)^{2} \neq 1(n=0,1,2, \ldots)$ and $r$ is an arbitrary fixed non-negative integer, then the system $\left\{\psi_{n}(x)\right\}(n=0,1, \ldots ; n \neq r)$ is minimal in $L_{2}(0,1)$.

Corollary $5([15])$. If $\left(b \lambda_{n}\right)^{2} \neq 1(n=0,1,2, \ldots)$ and $r$ is an arbitrary fixed non-negative integer, then the system $\left\{\varphi_{n}(x)\right\}$ which are biorthogonally conjugates to the system $\left\{\psi_{n}(x)\right\}$ is given by the following formula:

$$
\varphi_{n}(x)=a_{n}\left[\frac{\psi_{n}(1-x)+b \lambda_{n} \psi_{n}(x)}{\psi_{n}(1)}-\frac{\psi_{r}(1-x)+b \lambda_{r} \psi_{r}(x)}{\psi_{r}(1)}\right],
$$

where $a_{n}=-\left(\frac{1}{\lambda_{n}}+\lambda_{n}\left(a \frac{\partial \psi\left(1, \lambda_{n}\right)}{\partial \lambda}+b \frac{\partial \psi^{\prime}\left(1, \lambda_{n}\right)}{\partial \lambda}\right)\right)^{-1}$. 
Theorem 6 ( [15]). If $\left(b \lambda_{n}\right)^{2} \neq 1(n=0,1,2, \ldots)$ and $r$ is an arbitrary fixed nonnegative integer, then the system $\left\{\psi_{n}(x)\right\}(n=0,1, \ldots ; n \neq r)$ is a basis in $L_{p}(0,1)$ $(1<p<\infty)$, and this basis is unconditional for $p=2$.

\section{Main Results}

3.1. The Sharpened Asymptotics for Eigenparameters. The asymptotic estimates of eigenvalues (6) and eigenfunctions (7) of the problem (1)-(2) should be sharpened to give theorem about the uniform convergence conditions of Fourier series expansions of continuous functions in terms of eigenfunctions of this problem. In this subsection, the expression and proof of the relevant theorem will be given to finalize these asymptotic estimates.

Theorem 7. Let $\lambda_{n}=\mu_{n}^{2}\left(\operatorname{Re} \mu_{n} \geq 0\right)$. The asymptotic estimates

$$
\begin{gathered}
\mu_{n}=\left(n-\frac{1}{2}\right) \pi+\frac{c_{0}}{n \pi}+O\left(\frac{\delta_{n}}{n}\right) \\
\psi_{n}(x)=\frac{\sin \left(n-\frac{1}{2}\right) \pi x}{\left(n-\frac{1}{2}\right) \pi}+\frac{\alpha(x)}{(n \pi)^{2}} \cos \left(n-\frac{1}{2}\right) \pi x \\
+\frac{\alpha_{n}(x)}{2(n \pi)^{2}} \cos \left(n-\frac{1}{2}\right) \pi x+\frac{\beta_{n}(x)}{2(n \pi)^{2}} \sin \left(n-\frac{1}{2}\right) \pi x \\
+O\left(\frac{\delta_{n}}{n^{2}}\right)
\end{gathered}
$$

are valid for sufficiently large $n$, where $c_{0}=\frac{a}{b}+\frac{1}{2} \int_{0}^{1} q(\theta) d \theta, \alpha(x)=c_{0} x-\frac{1}{2} \int_{0}^{x} q(\theta) d \theta$, $\alpha_{n}(x)=\int_{0}^{x} q(\theta) \cos (2 n-1) \pi \theta d \theta, \beta_{n}(x)=\int_{0}^{x} q(\theta) \sin (2 n-1) \pi \theta d \theta$ and $\delta_{n}=\left|\int_{0}^{1} q(\theta) \cos (2 n-1) \pi \theta d \theta\right|+\frac{1}{n}$.

Proof. Let $\lambda=\lambda_{n}, \quad n \in \mathbb{N}$. The eigenvalues of the problem (1)-(2) are the roots of the equation

$$
\psi_{n}^{\prime}(0)=\lambda_{n}\left(a \psi_{n}(1)+b \psi_{n}^{\prime}(1)\right)
$$

because $\psi_{n}(x)$ is the solution of the equation (1) which satisfies the conditions (3).

Firstly, we now need to do the following calculations:

Let $\lambda_{n}=\mu_{n}^{2}$. Then, the estimate

$$
\mu_{n}=\sqrt{\lambda_{n}}=\left(n-\frac{1}{2}\right) \pi+\xi_{n}
$$

is satisfied from (6), where $\xi_{n}=O\left(n^{-1}\right)$. The main purpose of this proof is to write a sharper expression of the estimate $\xi_{n}$. 
On the other hand, it can be easily seen that the estimate

$$
\psi_{n}(x)=\frac{\sin \mu_{n} x}{\mu_{n}}+O\left(n^{-2}\right)
$$

is valid from 5 .

By using $(12)$ and 13 in the estimate obtained as a result of some basic calculations in (4) and in the differential of this estimate with respect to $x$, we obtain the estimates

$$
\begin{aligned}
\psi_{n}(x) & =\frac{\sin \mu_{n} x}{\mu_{n}}-\frac{\cos \left(n-\frac{1}{2}\right) \pi x}{2(n \pi)^{2}} \int_{0}^{x} q(\theta) d \theta \\
& +\frac{\cos \left(n-\frac{1}{2}\right) \pi x}{2(n \pi)^{2}} \int_{0}^{x} q(\theta) \cos (2 n-1) \pi \theta d \theta \\
& +\frac{\sin \left(n-\frac{1}{2}\right) \pi x}{2(n \pi)^{2}} \int_{0}^{x} q(\theta) \sin (2 n-1) \pi \theta d \theta+O\left(n^{-3}\right), \\
\psi_{n}^{\prime}(x) & =\cos \mu_{n} x-\frac{\sin \left(n-\frac{1}{2}\right) \pi x}{2 n \pi} \int_{0}^{x} q(\theta) d \theta \\
& -\frac{\sin \left(n-\frac{1}{2}\right) \pi x}{2 n \pi} \int_{0}^{x} q(\theta) \cos (2 n-1) \pi \theta d \theta \\
& +\frac{\cos \left(n-\frac{1}{2}\right) \pi x}{2 n \pi} \int_{0}^{x} q(\theta) \sin (2 n-1) \pi \theta d \theta+O\left(n^{-2}\right) .
\end{aligned}
$$

With the help of the calculations

$$
\begin{gathered}
\sin \mu_{n}=(-1)^{n-1}+O\left(n^{-2}\right), \quad \cos \mu_{n}=(-1)^{n} \xi_{n}+O\left(n^{-3}\right), \\
\frac{\sin \mu_{n}}{\mu_{n}}=\frac{(-1)^{n-1}}{n \pi}+O\left(n^{-4}\right)
\end{gathered}
$$

from (12), we can respectively rewrite for $x=1$ the estimates 14 and 15 as follows:

$$
\begin{gathered}
\psi_{n}(1)=\frac{(-1)^{n-1}}{n \pi}+O\left(n^{-2}\right), \\
\psi_{n}^{\prime}(1)=(-1)^{n} \xi_{n}+\frac{(-1)^{n-1}}{2 n \pi} \int_{0}^{x} q(\theta) d \theta+O\left(\frac{\delta_{n}}{n^{2}}\right),
\end{gathered}
$$

where $\delta_{n}=\left|\int_{0}^{1} q(\theta) \cos (2 n-1) \pi \theta d \theta\right|+\frac{1}{n}$. 
Secondly, by considering (16) and (17) in the equation (11), we obtain the equation

$$
\frac{a(-1)^{n-1}}{n \pi}+b(-1)^{n} \xi_{n}+\frac{b(-1)^{n-1}}{2 n \pi} \int_{0}^{1} q(\theta) d \theta+O\left(\frac{\delta_{n}}{n}\right)=0 .
$$

The last equation implies that the estimate

$$
\xi_{n}=\frac{\frac{a}{b}+\frac{1}{2} \int_{0}^{1} q(\theta) d \theta}{n \pi}+O\left(\frac{\delta_{n}}{n}\right)
$$

holds. The above formal reasoning shows that the estimate (9) should hold.

Furthermore, by using (9) and (18), we have the term

$$
\frac{\sin \mu_{n} x}{\mu_{n}}=\frac{\sin \left(n-\frac{1}{2}\right) \pi x}{\left(n-\frac{1}{2}\right) \pi}+\frac{c_{0} x}{(n \pi)^{2}} \cos \left(n-\frac{1}{2}\right) \pi x+O\left(\frac{\delta_{n}}{n^{2}}\right)
$$

where $c_{0}=\frac{a}{b}+\frac{1}{2} \int_{0}^{1} q(\theta) d \theta$.

Consequently, the asymptotic estimate (10) follows from (14) and 19). The Theorem 7 is proved with this.

3.2. The Uniform Convergence Conditions of Spectral Expansions. In this section, we will investigate the uniform convergence of Fourier series expansions for the continuous functions in the system of eigenfunctions and the functions which are biorthogonally conjugate to eigenfunctions of the problem (1)-(2)

Theorem 8. Let $r$ be an arbitrary fixed non-negative integer. If $f(x) \in C[0,1]$ has a uniformly convergent Fourier series expansion in the system $\left\{\sqrt{2} \sin \left(n-\frac{1}{2}\right) \pi x\right\}_{n=1}^{\infty}$ on $[0,1]$, then this function can be expanded as Fourier series in the system $\left\{\psi_{n}(x)\right\}$ $(n=0,1, \ldots ; n \neq r)$ on $[0,1]$ and this expansion are uniformly convergent on the same interval.

Proof. Let us consider the Fourier series expansion of a continuous function $f(x)$ in the system $\left\{\psi_{n}(x)\right\}$ on $[0,1]$ :

$$
\Psi(x)=\sum_{n=0, n \neq r}^{\infty}\left(f, \varphi_{n}\right) \psi_{n}(x),
$$

where the system $\varphi_{n}(x)(n=0,1, \ldots ; n \neq r)$ is defined by (8). In addition, the estimate $a_{n}$ in this system can also be written as the form

$$
a_{n}=\frac{2(-1)^{n-1}}{b n \pi}+O\left(n^{-2}\right) \text {. }
$$


Now, we can analyze the series 20 in the form

$$
F(x)=\sum_{n=r+1}^{\infty}\left(f, \varphi_{n}\right) \psi_{n}(x)=\sum_{i=1}^{3} F_{i}(x)
$$

to investigate the uniform convergence of it on $[0,1]$, where

$$
\begin{gathered}
F_{1}(x)=\sum_{n=r+1}^{\infty} \frac{a_{n}}{\psi_{n}(1)}\left(f, \overline{\psi_{n}(1-x)}\right) \psi_{n}(x), \\
F_{2}(x)=\sum_{n=r+1}^{\infty} \frac{b a_{n} \lambda_{n}}{\psi_{n}(1)}\left(f, \overline{\psi_{n}(x)}\right) \psi_{n}(x), \\
F_{3}(x)=\frac{\left(f, \overline{\psi_{r}(1-x)+b \lambda_{r} \psi_{r}(x)}\right)}{\psi_{r}(1)} \sum_{n=r+1}^{\infty} a_{n} \psi_{n}(x) .
\end{gathered}
$$

Firstly, the series 23 is uniformly convergent on the interval $[0,1]$ by virtue of the estimate

$$
\frac{a_{n}}{\psi_{n}(1)}=\frac{2}{b}+O\left(n^{-1}\right),
$$

which follows from (16) and (21).

Secondly, let us show that the series $(24)$ is uniformly convergent on the interval $[0,1]$. Thus, we consider the sequence $\left\{S_{m}\right\}_{m=1}^{\infty}$, which is defined the partial sums of the series (24) can be represented in the form

$$
S_{m}(x)=\sum_{n=r+1}^{m} \frac{b a_{n} \lambda_{n}}{\psi_{n}(1)}\left(f, \overline{\psi_{n}(x)}\right) \psi_{n}(x)
$$

On the other hand, since $\frac{b a_{n} \lambda_{n}}{\psi_{n}(1)}=2 \lambda_{n}+O(n)$ by using 26 , we have the identity

$$
\begin{aligned}
\sqrt{2} \mu_{n} \psi_{n}(x) & =\sqrt{2} \sin \left(n-\frac{1}{2}\right) \pi x+\frac{\sqrt{2} \alpha(x)}{n \pi} \cos \left(n-\frac{1}{2}\right) \pi x \\
& +\frac{\alpha_{n}(x)}{\sqrt{2} n \pi} \cos \left(n-\frac{1}{2}\right) \pi x+\frac{\beta_{n}(x)}{\sqrt{2} n \pi} \sin \left(n-\frac{1}{2}\right) \pi x+O\left(\frac{\delta_{n}}{n}\right),
\end{aligned}
$$

where $\alpha(x), \alpha_{n}(x)$ and $\beta_{n}(x)$ are defined on Theorem 7 and $\lambda_{n}=\mu_{n}^{2}$. From the last identity, we obtain

$$
\frac{b a_{n} \lambda_{n}}{\psi_{n}(1)}\left(f, \overline{\psi_{n}(x)}\right) \psi_{n}(x)=\left(f, \sqrt{2} \sin \left(n-\frac{1}{2}\right) \pi x\right) \sqrt{2} \sin \left(n-\frac{1}{2}\right) \pi x+G_{n}(x),
$$


where

$$
\begin{aligned}
G_{n}(x) & =\left(f, \sin \left(n-\frac{1}{2}\right) \pi x\right) O\left(n^{-1}\right) \\
& +\left(f(x) \alpha(x), \cos \left(n-\frac{1}{2}\right) \pi x\right) O\left(n^{-1}\right) \\
& +\left(f, \alpha_{n}(x) \cos \left(n-\frac{1}{2}\right) \pi x\right) O\left(n^{-1}\right) \\
& +\left(f, \beta_{n}(x) \sin \left(n-\frac{1}{2}\right) \pi x\right) O\left(n^{-1}\right) \\
& +O\left(\frac{\delta_{n}}{n}\right) .
\end{aligned}
$$

So, the identity

$$
S_{m}(x)=\sum_{n=r+1}^{m}\left(f, \sqrt{2} \sin \left(n-\frac{1}{2}\right) \pi x\right) \sqrt{2} \sin \left(n-\frac{1}{2}\right) \pi x+\sum_{n=r+1}^{m} G_{n}(x)
$$

holds.

The first sum in the last identity is the Fourier series expansion of $f(x)$ in the system $\left\{\sqrt{2} \sin \left(n-\frac{1}{2}\right) \pi x\right\}_{n=1}^{\infty}$. Consequently, the uniform convergence of this sum as $m \rightarrow \infty$ on the interval $[0,1]$ is stipulated in the assumptions of the Theorem 8 .

On the other hand, we now will examine the uniformly convergence of the other sums

$$
\sum_{n=r+1}^{m} G_{n}(x)
$$

as $m \rightarrow \infty$. From (27), we have

$$
\begin{aligned}
\left|G_{n}(x)\right| & \leq \frac{c_{1}}{n}\left\{\left|\left(f, \sin \left(n-\frac{1}{2}\right) \pi x\right)\right|+\left|\left(f(x) \alpha(x), \cos \left(n-\frac{1}{2}\right) \pi x\right)\right|\right. \\
& +\left|\left(f, \alpha_{n}(x) \cos \left(n-\frac{1}{2}\right) \pi x\right)\right|+\left|\left(f, \beta_{n}(x) \sin \left(n-\frac{1}{2}\right) \pi x\right)\right| \\
& \left.+O\left(\delta_{n}\right)\right\} \\
& \leq c_{2}\left\{\left|\left(f, \sin \left(n-\frac{1}{2}\right) \pi x\right)\right|^{2}+\left|\left(f(x) \alpha(x), \cos \left(n-\frac{1}{2}\right) \pi x\right)\right|^{2}\right. \\
& +\left(\int_{0}^{1}\left|f(x) \alpha_{n}(x)\right| d x\right)^{2}+\left(\int_{0}^{1}\left|f(x) \beta_{n}(x)\right| d x\right)^{2} \\
& \left.+O\left(\frac{\delta_{n}}{n}\right)\right\}
\end{aligned}
$$

for sufficiently large $n$, where $c_{1}$ and $c_{2}$ are real constants. 
The sums

$$
\begin{aligned}
\sum_{n=r+1}^{m}\left|\left(f, \sin \left(n-\frac{1}{2}\right) \pi x\right)\right|^{2} & \sum_{n=r+1}^{m}\left|\left(f(x) \alpha(x), \cos \left(n-\frac{1}{2}\right) \pi x\right)\right|^{2}, \\
& \sum_{n=r+1}^{m} O\left(\frac{\delta_{n}}{n}\right)
\end{aligned}
$$

are convergent as $m \rightarrow \infty$. Moreover, by the Bessel inequality, we get

$$
\begin{aligned}
\sum_{n=r+1}^{\infty}\left(\int_{0}^{1}\left|f(x) \beta_{n}(x)\right| d x\right)^{2} & \leq\|f\|^{2} \sum_{n=r+1}^{\infty} \int_{0}^{1}\left|\beta_{n}(x)\right|^{2} d x \\
& =\|f\|^{2} \int_{0}^{1} \sum_{n=r+1}^{\infty}\left|\int_{0}^{x} q(\theta) \sin (2 n-1) \pi \theta d \theta\right|^{2} d x \\
& \leq c_{3}\|f\|^{2} \int_{0}^{1} \int_{0}^{x}|q(\theta)|^{2} d \theta d x \\
& \leq c_{3}\|f\|^{2}\|q\|^{2}
\end{aligned}
$$

and, similarly

$$
\sum_{n=r+1}^{\infty}\left(\int_{0}^{1}\left|f(x) \alpha_{n}(x)\right| d x\right)^{2} \leq c_{4}\|f\|^{2}\|q\|^{2},
$$

where $c_{3}$ and $c_{4}$ are real constants. Namely, the series 28 as $m \rightarrow \infty$ is absolutely and uniformly convergent on the interval $[0,1]$.

Thirdly, the series 25 is uniformly convergent on the interval $[0,1]$, by virtue of the estimate

$$
\sum_{n=r+1}^{\infty} a_{n} \psi_{n}(x)=\sum_{n=r+1}^{\infty} O\left(n^{-2}\right)
$$

which follows from (14) and 21.

As a result of all these calculations, the series 222 , so the series 20$]$, is uniformly convergent on the interval $[0,1]$. The proof of the Theorem 8 is completed.

Corollary 9. If $f(x)$ is a function that provides the hypothesis of the Theorem 8 , then this function can also be expanded as Fourier series in the biorthogonal system $\varphi_{n}(x)(n=0,1, \ldots ; n \neq r)$ is defined by (8) on $[0,1]$ and this expansion are uniformly convergent on this interval.

Proof. Consider the Fourier series of $f(x)$ in the system $(8)$ on $[0,1]$ :

$$
\Phi(x)=\sum_{n=0, n \neq r}^{\infty}\left(f, \psi_{n}\right) \varphi_{n}(x) \approx \sum_{n=r+1}^{\infty}\left(f, \psi_{n}\right) \varphi_{n}(x)=\sum_{i=1}^{3} H_{i}(x),
$$


where

$$
\begin{gathered}
H_{1}(x)=\sum_{n=r+1}^{\infty} \frac{a_{n}}{\psi_{n}(1)}\left(f, \overline{\psi_{n}(x)}\right) \psi_{n}(1-x), \\
H_{2}(x)=\sum_{n=r+1}^{\infty} \frac{b a_{n} \lambda_{n}}{\psi_{n}(1)}\left(f, \overline{\psi_{n}(x)}\right) \psi_{n}(x), \\
H_{3}(x)=-\frac{\psi_{r}(1-x)+b \lambda_{r} \psi_{r}(x)}{\psi_{r}(1)} \sum_{n=r+1}^{\infty} a_{n}\left(f, \overline{\psi_{n}(x)}\right) .
\end{gathered}
$$

It can be seen that the convergence of the series (29)-(31) are calculated by a similar method using in examining the convergence of the series (23)-25).

Authors Contribution Statement The authors contributed equally. All authors read and approved the final copy of the manuscript.

Declaration of Competing Interests The authors declare that they have no competing interest.

Acknowledgement The authors thank the referees for their valuable comments and constructive suggestions.

\section{REFERENCES}

[1] Gulyaev, D. A., On the uniform convergence of spectral expansions for a spectral problem with boundary conditions of the third kind one of which contains the spectral parameter, Differential Equations, 47(10) (2011), 1520-1524.

[2] Gulyaev, D. A., On the convergence in $W_{2}^{m}$ of spectral expansions for a spectral problem with boundary conditions of the third kind one of which contains the spectral parameter, Differential Equations, 48(10) (2012), 1429-1432.

[3] Kapustin, N. Yu., On the uniform convergence of the Fourier series for a spectral problem with squared spectral parameter in a boundary condition, Differential Equations, 46(10) (2010), $1507-1510$.

[4] Kapustin, N. Yu., On the uniform convergence in $C^{1}$ of Fourier series for a spectral problem with squared spectral parameter in a boundary condition, Differential Equations, 47(10) (2011), 1408-1413.

[5] Kapustin, N. Yu., On the spectral problem arising in the solution of a mixed problem for the heat equation with a mixed derivative in the boundary conditions, Differential Equations, 48(5) (2012), 701-706.

[6] Kapustin, N. Yu. and Moiseev, E. I., Convergence of spectral expansions for functions of the Hölder class for two problems with a spectral parameter in the boundary condition, Differential Equations, 36(8) (2000), 1182-1188.

[7] Kapustin, N. Yu. and Moiseev, E. I., A remark on the convergence problem for spectral expansions corresponding to a classical problem with spectral parameter in the boundary condition, Differential Equations, 37(12) (2001), 1677-1683.

[8] Kerimov, N. B. and Maris E. A., On the basis properties and convergence of expansions in terms of eigenfunctions for a spectral problem with a spectral parameter in the boundary condition, Proc. IMM of NAS, 40 (2014), 245-258. 
[9] Kerimov, N. B. and Maris E. A., On the uniform convergence of the Fourier Series for one spectral problem with a spectral parameter in a boundary condition, Math. Methods Appl. Sci, 39(9) (2016), 2298-2309.

[10] Kerimov, N. B. and Maris E. A., On the uniform convergence of Fourier series expansions for Sturm-Liouville problems with a spectral parameter in the boundary conditions, Results in Mathematics, 73(3) (2018), 102.

[11] Koyunbakan, H., Solving the hyperbolic problem obtained by transmutation operator, Leading-Edge Research on Evolution Equations, (2008), 185-195.

[12] Koyunbakan, H., The inverse nodal problem for a differential operator with an eigenvalue in the boundary condition, Applied Mathematics Letters, 21(12) (2008), 1301-1305.

[13] Levitan, B. M. and Sargsjan, I. S., Sturm-Liouville and Dirac Operators, Kluwer Academic Publishers: Netherlands, 1991.

[14] Marchenkov, D. B., On the convergence of spectral expansions of functions for problems with a spectral parameter in a boundary condition, Differential Equations, 41(10) (2005), 1496-1500.

[15] Maris E. A. and Goktas, S., On the spectral properties of a Sturm-Liouville problem with eigenparameter in the boundary condition, HJMS, 49(4) (2020), 1373-1382.

[16] Tikhonov, A. N. and Samarskii A. A., Equations of Mathematical Physics, New York, Courier Corporation, 2013; [in russian] Uravneniya Matematicheskoi Fiziki, Moscow, 1972.

[17] Wang, Y. P. and Koyunbakan, H., On the Hochstadt-Lieberman theorem for discontinuous boundary-valued problems, Acta Mathematica Sinica, English Series, 30(6) (2014), 985-992. 